\title{
Blue light-induced apoptosis of human promyelocytic leukemia cells via the mitochondrial-mediated signaling pathway
}

\author{
JIANJIAN ZHUANG ${ }^{1 *}$, YANGE LIU ${ }^{2 *}$, QINGXIA YUAN ${ }^{2 *}$, \\ JUNSONG LIU ${ }^{1}$, YAN LIU ${ }^{2}$, HONGDONG LI ${ }^{1}$ and DI WANG ${ }^{2}$ \\ ${ }^{1}$ State Key Laboratory of Superhard Materials; ${ }^{2}$ School of Life Sciences, Jilin University, Changchun, Jilin 130012, P.R. China
}

Received September 25, 2017; Accepted February 28, 2018

DOI: $10.3892 / \mathrm{ol} .2018 .8162$

\begin{abstract}
Acute promyelocytic leukemia is frequently associated with dizziness, fever, nausea, hematochezia and anemia. Blue light, or light with wavelengths of 400-480 nm, transmits high levels of energy. The aim of the present study was to determine the pro-apoptotic effects of blue light (wavelength, $456 \mathrm{~nm}$; radiation power, $0.25 \mathrm{~mW} / \mathrm{cm}^{2}$ ) and the underlying mechanisms in a human promyelocytic leukemia cell line (HL60). Blue light reduced the viability and enhanced the mortality of HL60 cells in a time-dependent manner. Exposure to blue light for $24 \mathrm{~h}$ caused depolarization of the mitochondrial membrane potential and the overproduction of reactive oxygen species in HL60 cells. In a nude mouse model, 9-day exposure to blue light markedly suppressed the growth of HL60-xenografted tumors; however, it had no effect on hepatic and renal tissues. In addition, blue light abrogated the expression of B-cell lymphoma (Bcl)-2 and Bcl extra-long, while enhancing the levels of $\mathrm{Bcl}-2$-associated $\mathrm{X}$ protein, cytochrome $c$, and cleaved caspases-3 and -9 in tumor tissues. The results suggested that the pro-apoptotic effects of blue light in human promyelocytic leukemia cells may be associated with the mitochondrial apoptosis signaling pathway.
\end{abstract}

\section{Introduction}

Acute promyelocytic leukemia (APL), a subtype of acute myeloid leukemia (AML), accounts for $46 \%$ of leukemia cases and is frequently associated with dizziness, fever,

Correspondence to: Dr Di Wang, School of Life Sciences, Jilin University, 2699 Qianjin Street, Changchun, Jilin 130012, P.R. China E-mail: jluwangdi@outlook.com

Professor Hongdong Li, State Key Laboratory of Superhard Materials, Jilin University, 2699 Qianjin Street, Changchun, Jilin 130012, P.R. China

E-mail: hdli@jlu.edu.cn

*Contributed equally

Key words: blue light, leukemia, HL60 cells, apoptosis, mitochondrion nausea, hematochezia and anemia $(1,2)$. Currently, the standard treatment for APL is combination chemotherapy, which may cause gastrointestinal effects, multi-organ impairment and alopecia. Therefore, new therapeutic options for APL are urgently needed. Near-infrared light can easily penetrate the skin and is absorbed deeply into tissues (3). Accordingly, the ability of a near-infrared (NIR) laser and hollow gold nanospheres (HAuNSs) to modulate the release of anticancer agents was tested using the antitumor drug paclitaxel, which exhibits surface plasmon absorbance in the NIR region and significant anti-tumor effects, demonstrated strong cytotoxicity and reduced tumorigenesis in nude mice (4). Another study observed survival rates no higher than $30 \%$ among human lung carcinoma epithelial cells (A549) exposed to photodynamic therapy for $4 \mathrm{~h}$ (5). Recently, our group has successfully confirmed that blue light can enhance the proliferation inhibition of HL60 cells treated by all-trans retinoic acid/nanodiamonds (6). However, the pro-apoptotic effects of blue light on promyelocytic leukemia cells and related mouse models, as well as the possible mechanisms, have not yet been reported.

Pro-apoptotic factors activate mitochondrial apoptotic signaling pathways, which lead to the excessive opening of mitochondrial permeability transition pores (MPTPs). This excessive pore opening causes the dissipation of mitochondrial membrane potential (MMP) across the inner mitochondrial membrane, efflux of $\mathrm{Ca}^{2+}$, uncoupling of the respiratory chain and production of superoxide $(7,8)$. Subsequently, these changes enhance the release of cytochrome $c$, apoptosis-inducing factor and B-cell lymphoma-2 (Bcl-2) family proteins from the mitochondria into the membrane space, thus triggering the cell to enter apoptosis.

Bcl-2 family proteins localize within the mitochondria and appear to regulate mitochondrial outer membrane permeability by binding to mitochondrial channels (9). Bcl-2-associated X protein (Bax) induces the cytoplasmic release of mitochondrial mediators such as cytochrome $c$, which serves as a marker of mitochondrial injury and is involved in the caspase-dependent apoptosis pathway (10). Moreover, the mitochondrion is the major site of reactive oxygen species (ROS) generation (11). Apoptosis can be triggered both by ROS-induced abnormal gene expression and the blockage of cell communication (12).

This study aimed to investigate the potential systematic effects of blue light therapy for APL. Using in vitro and in vivo 
experiments, we successfully explored the pro-apoptotic effects of blue light and the underlying mechanisms related to the mitochondrial apoptotic pathway.

\section{Materials and methods}

Cell culture. HL60 human promyelocytic leukemia cells (American Type Culture Collection, Manassas, VA, USA) were cultured in Roswell Park Memorial Institute (RPMI)-1640 medium supplemented with $10 \%$ fetal bovine serum (FBS), $100 \mathrm{U} / \mathrm{ml}$ penicillin and $100 \mu \mathrm{g} / \mathrm{ml}$ streptomycin in a humidified atmosphere containing $5 / 95 \% \mathrm{CO}_{2} /$ air at $37^{\circ} \mathrm{C}$. All cell culture reagents were obtained from Invitrogen (Thermo Fisher Scientific, Inc., Waltham, MA, USA).

Cell viability assessment. Cells were exposed to blue light (wavelength: $456 \mathrm{~nm}$; storage battery power supply: $12 \mathrm{~V}$; radiation power: $0.25 \mathrm{~mW} / \mathrm{cm}^{2}$ (Jiangmen Weigu Lighting Technology Co., Ltd., Jiangmen, China) for $10-56 \mathrm{~h}$ and to $50 \mu \mathrm{M}$ of PTX (Jiangsu Yew Pharmaceutical Co., Ltd., Jiangsu, China) for $24 \mathrm{~h}$. Cell viability was analyzed using a quantitative colorimetric assay and the Cell Counting Kit-8 (CCK-8; BestBio, Shanghai, China) according to the manufacturer's instructions.

Lactate dehydrogenase (LDH) release assessment. Cells were exposed to blue light and $50 \mu \mathrm{M}$ of PTX for $24 \mathrm{~h}$, after which the concentrations of LDH in the culture supernatants were detected using an in vitro Toxicology Assay kit (C0017; Beyotime Biotechnology, Jiangsu, China). Data were expressed in terms of cell mortality, according to the instruction manual.

Cell apoptosis assessment. Cells were exposed to blue light and $50 \mu \mathrm{M}$ of PTX for $24 \mathrm{~h}$. Subsequently, suspended cells were incubated with Annexin V-FITC and propidium iodide for $10 \mathrm{~min}$ at $25^{\circ} \mathrm{C}$ in darkness, and then analyzed using a Muse $^{\mathrm{TM}}$ Cell Analyzer (EMD Millipore, Billerica, MA, USA) according to the manufacturer's instructions.

MMP assessment. Cells were exposed to blue light and $50 \mu \mathrm{M}$ of PTX for $24 \mathrm{~h}$, and subsequently incubated with $2 \mu \mathrm{M}$ of 5,5', 6,6'-tetrachloro-1,1', 3,3'tetraethylbenzimidazol-ylcarbocyanine iodide (JC-1; Sigma-Aldrich; Merck KGaA, Darmstadt, Germany) for $20 \mathrm{~min}$ at $25^{\circ} \mathrm{C}$ in darkness. Changes in the levels of red and green fluorescence were detected using a Muse $^{\mathrm{TM}}$ Cell Analyzer (EMD Millipore).

ROS assessment. Cells were exposed to blue light and $50 \mu \mathrm{M}$ of PTX for $24 \mathrm{~h}$, followed by $10 \mu \mathrm{M}$ of 2'-7'-dichlorodihydrofluorescein diacetate (DCFH-DA; Sigma-Aldrich; Merck $\mathrm{KGaA})$ at $37^{\circ} \mathrm{C}$ for $10 \mathrm{~min}$ in darkness. Changes in the levels of green fluorescence were detected using a Muse ${ }^{\mathrm{TM}}$ Cell Analyzer (EMD Millipore).

HL60-xenografted tumor mouse model. The animal experimental protocol was approved by the Animal Ethics Committee of Jilin University, and all experiments were performed in accordance with the National Institutes of Health Guide for the Care and Use of Laboratory Animals (NIH Publications no. 8023, 1978 revision). Five-week-old male BALB/c nude mice (Vital River Laboratory Animal Technology Co., Ltd., Beijing, China) were housed in groups of three in cages exposed to a 12-h light/dark cycle (lighted from 7:00 a.m. to 7:00 p.m.) at $23 \pm 1^{\circ} \mathrm{C}$. Water and food were available ad libitum.

HL60 cells were harvested from mid-log-phase cultures, and $0.1 \mathrm{ml}$ of cell suspension $\left(1 \times 10^{8}\right.$ cells $\left./ \mathrm{ml}\right)$ was subcutaneously (s.c.) injected into the right abdomen of each mouse. When the tumor diameters reached 3-5 $\mathrm{mm}$, the mice were randomly divided into two groups $(\mathrm{n}=3)$ and exposed to normal (CTRL) or blue light for nine days. The body weights and tumor dimensions were measured daily, and the tumor volumes $\left(\mathrm{mm}^{3}\right)$ were calculated as follows: Length $\mathrm{x}(\text { width })^{2} \mathrm{x} 0.5$. Finally, the mice were sacrificed via injection with $200 \mathrm{mg} / \mathrm{kg}$ of pentobarbital, after which the tumors were dissected and liver and kidney tissues were collected.

Histopathological study. Hematoxylin and eosin (H\&E) staining was used to assess hepatic and renal histology. Briefly, liver and kidney tissues were fixed in $4 \%$ paraformaldehyde for $24 \mathrm{~h}$, and subsequently dehydrated in a stepwise manner using an ethanol gradient $(50,70,80,90,95$ and 100\%). The dehydrated samples were immersed in xylene for $30 \mathrm{~min}$ and incubated overnight in paraffin at $65^{\circ} \mathrm{C}$. Once embedded, the specimens were cut into $4-\mu \mathrm{m}$-thick slices using a microtome (Leica, Wetzlar, Germany) and placed on microscopy slides. The sections were deparaffinized with fresh xylene for $10 \mathrm{~min}$, hydrated using an ethanol gradient (100, 90, 80 and 70\%) and washed thrice with distilled/deionized water. After staining with $\mathrm{H} \& \mathrm{E}$, the sections were examined using an IX73 inverted microscope with a x40 objective (Olympus, Tokyo, Japan).

Western blot. Tumor tissues were lysed using RIPA buffer (Sigma-Aldrich; Merck KGaA) containing a $1 \%$ protease inhibitor cocktail (Sigma-Aldrich; Merck KGaA) and 2\% PMSF (Sigma-Aldrich; Merck KGaA). Forty micrograms of protein per sample were separated on a $12 \%$ SDS-PAGE gel. The proteins were then transferred electrophoretically to $0.45 \mu \mathrm{m}$ nitrocellulose membranes, which were then incubated overnight with primary antibodies against $\mathrm{Bcl}-2, \mathrm{Bax}, \mathrm{Bcl}$ extra-long (Bcl-xL), cytochrome $c$, cleaved caspases-3 and -9 and glyceraldehyde-3-phosphate dehydrogenase (GAPDH) (Cell Signaling Technology, Inc., Danvers, MA, USA) at $4^{\circ} \mathrm{C}$ (all dilutions: 1:1,000). Subsequently, the membranes were incubated with horseradish peroxidase-conjugated secondary antibodies (Santa Cruz Biotechnology, Inc., Dallas, TX, USA) at room temperature for $4 \mathrm{~h}$. An enhanced chemiluminescence detection kit (GE Healthcare, Little Chalfont, UK) was then used to detect the labeled protein bands. The band intensities were quantified using Image $\mathbf{J}$ software (National Institutes of Health, Bethesda, MD, USA).

Statistical analysis. The data are expressed as means \pm standard deviations (SD). Statistical significance was detected using a one-way variance analysis (ANOVA), followed by Dunn's test, using SPSS version 16.0 software (SPSS, Inc., Chicago, IL, USA). P $<0.05$ was considered to indicate a statistically significant difference.

\section{Results}

Blue light induced apoptosis in HL60 cells. We first determined the optimal experimental blue light dose by controlling 
the exposure duration. Blue light reduced the viability of HL60 cells in a time-dependent manner from 12 to $56 \mathrm{~h}$ of exposure $(\mathrm{P}<0.001$; Fig. 1A). Both PTX and a 24-h exposure to blue light reduced cell viability by $>50 \%$ ( $\mathrm{P}<0.001$; Fig. $1 \mathrm{~B})$. The cell mortality rate was determined by $\mathrm{LDH}$ release, which was enhanced significantly in HL60 cells exposed to blue light for $24 \mathrm{~h}$ (Fig. 1C).

Apoptosis rates of 81.8 and $68.3 \%$ were observed among cells exposed to PTX $(50 \mu \mathrm{M})$ and blue light, respectively, for $24 \mathrm{~h}$ (Fig. 2A). Moreover, compared with the control cells, PTX or blue light treatment led to MMP depolarization rates of $72.5 \%$ (63.65 vs. $17.45 \%$ ) and $65.6 \%$ (50.85 vs. $17.45 \%)$ (Fig. 2B), which further promoted the apoptosis of HL60 cells.

In addition, ROS-mediated late mitochondrial events (13) were found to be strongly enhanced in HL60 cells exposed to blue light and PTX treatment (Fig. 2C).

Blue light suppressed tumor growth in nude mice. Blue light exhibited significant antitumor activity against HL60-xenografted tumors in a nude mouse model. A 9-day exposure to blue light strongly suppressed tumor growth without affecting the body weights of the mice, indicating that blue light has few side effects in normal tissues (Fig. 3A-C). A histopathological study in which few pathologic changes were observed between the livers and kidneys of blue-light exposed and non-exposed mice further confirmed the safety of blue light therapy (Fig. 3E and F). Sixteen days after tumor implantation, the tumors began to grow rapidly in the non-treated control mice, reaching a mean tumor volume of $975.5 \pm 283.4 \mathrm{~mm}^{3}$. In contrast, tumor growth was strongly inhibited in blue light-treated mice, beginning on day 7 ( $\mathrm{P}<0.05$; Fig. 3D).

Blue light regulated the expression of pro-and anti-apoptotic proteins. Compared with the non-treated mice, the tumor tissues of the blue light-treated mice exhibited significantly reduced expression of $\mathrm{Bcl}-2$ and $\mathrm{Bcl}-\mathrm{xL}$ and enhanced expression of Bax, cytochrome $c$ and cleaved caspases-3 and -9 $(\mathrm{P}<0.05$; Fig. 4). The $\mathrm{Bcl}-2 / \mathrm{Bax}$ ratio was $13.8 \%$ higher in the tumor tissues of the blue light-exposed mice, compared to the non-exposed mice (Fig. 4).

\section{Discussion}

In the present study, we have clarified the pro-apoptotic effects of blue light therapy and the underlying mechanisms in HL60 cells. We selected a 24-h exposure duration to investigate the mechanism underlying blue light-induced apoptosis in these cells. LDH is enriched in the cytoplasm and normally cannot pass through the membrane; however, cell damage causes a release of LDH into the extracellular space (14). We observed that blue light reduced the viability of HL60 cells in a time-dependent manner from 12 to $56 \mathrm{~h}$, as indicated by the release of $\mathrm{LDH}$.

A normal MMP is required to maintain oxidative phosphorylation and adenosine triphosphate (ATP) formation (7). A stable MMP thus confers a survival advantage upon the cell. Changes characteristic of mitochondrial apoptosis are commonly observed during cell apoptosis, which is characterized by the disruption of the MMP (15). In our study, blue light treatment caused depolarization of the MMP and thus
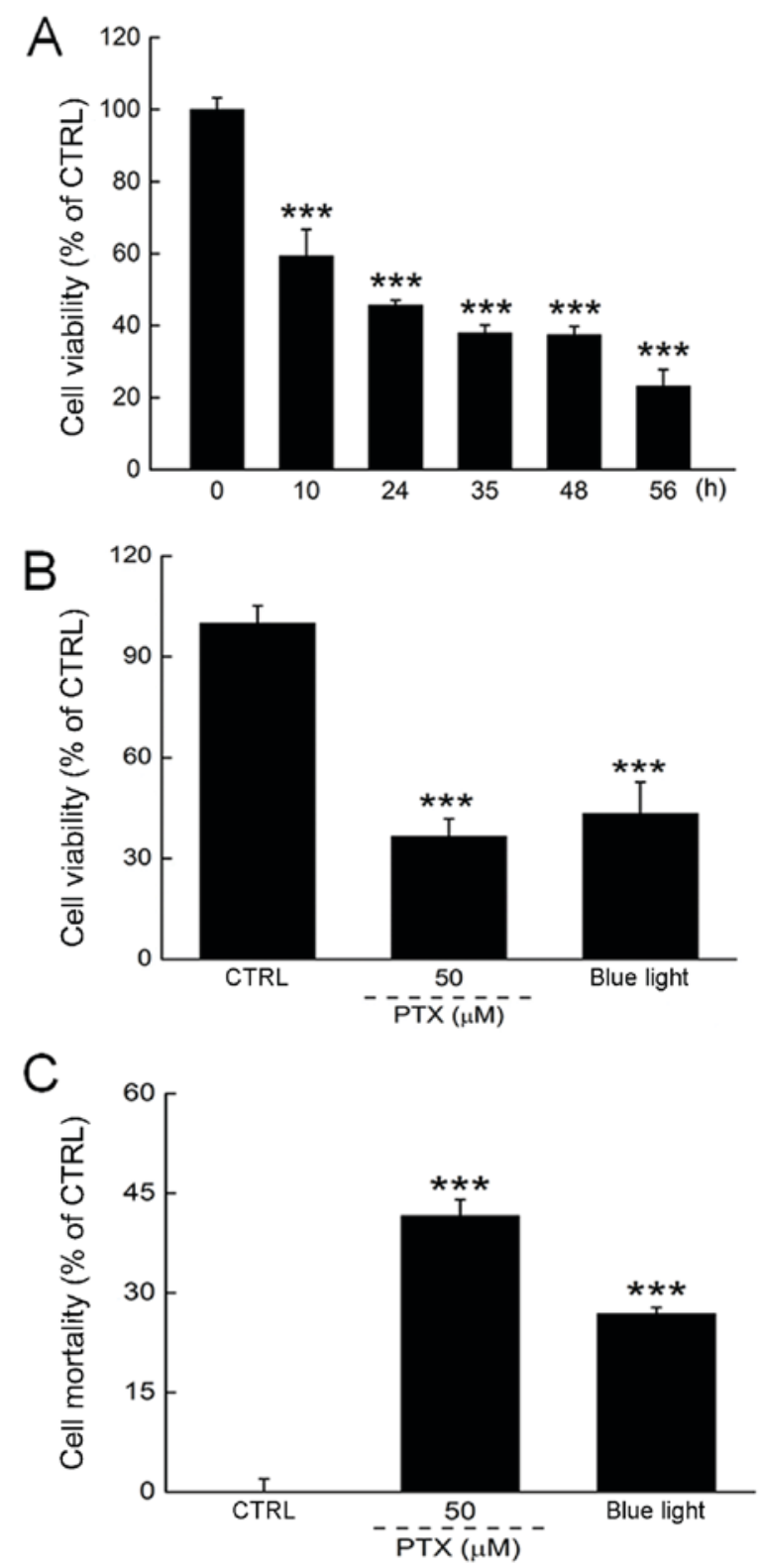

Figure 1. (A) Blue light reduced the cell viability of HL60 cells in a time-dependent manner. (B) 24-h exposure to PTX and blue light markedly reduced cell viability in HL60 cells. (C) 24-h exposure to PTX and blue light enhanced cell mortality, indicated by the enhanced release of lactate dehydrogenase. Data are expressed as a percentage of the corresponding control cells and the mean \pm standard deviation $(n=6) .{ }^{* * * *} \mathrm{P}<0.001$ vs. control cells. PTX, paclitaxel; CTRL, control.

promoted HL60 cell apoptosis. This process occurred before deoxyribonucleic acid fragmentation. As reported previously, ROS and mitochondria are linked by a short feedback loop as indicated by the cytoplasmic overproduction of ROS during mitochondria-dependent apoptosis, which further promotes the opening of the MPTP $(16,17)$. Our data suggest that blue light-mediated apoptosis in HL60 cells may involve the ROS-mediated mitochondrial apoptosis pathway.

Moreover, our in vivo data are consistent with those of in vitro investigations. The antitumor activity of blue light was further confirmed by our finding that blue light exposure strongly suppressed tumor growth without affecting the body weights of mice. Caspase-3, an important effector related to 

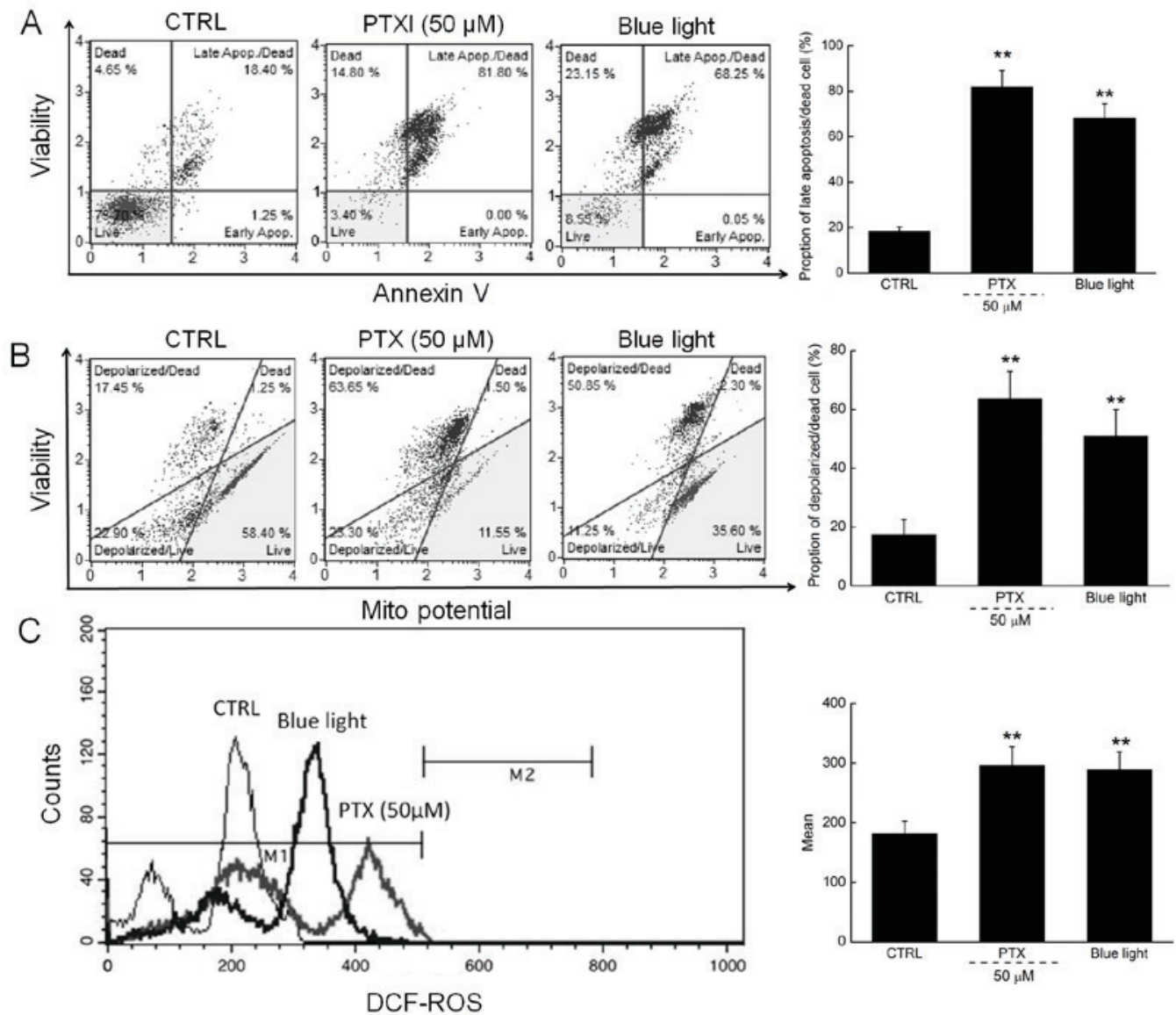

Figure 2. (A) 24-h exposure to PTX and blue light markedly enhanced the apoptotic rate of HL60 cells. (B) PTX and blue light induced the dissipation of mitochondrial membrane potential, detected via JC-1 staining. (C) 24-h exposure to PTX and blue light significantly enhanced intracellular ROS levels in HL60 cells. Data are expressed as the mean \pm standard deviation $(n=6) .{ }^{* *} \mathrm{P}<0.01$ vs. control cells. PTX, paclitaxel; ROS, reactive oxygen species; JC-1, 5,5', 6,6'-tetrachloro-1,1'3,3'tetraethylbenzimidazol-ylcarbocyanine iodide; CTRL, control.
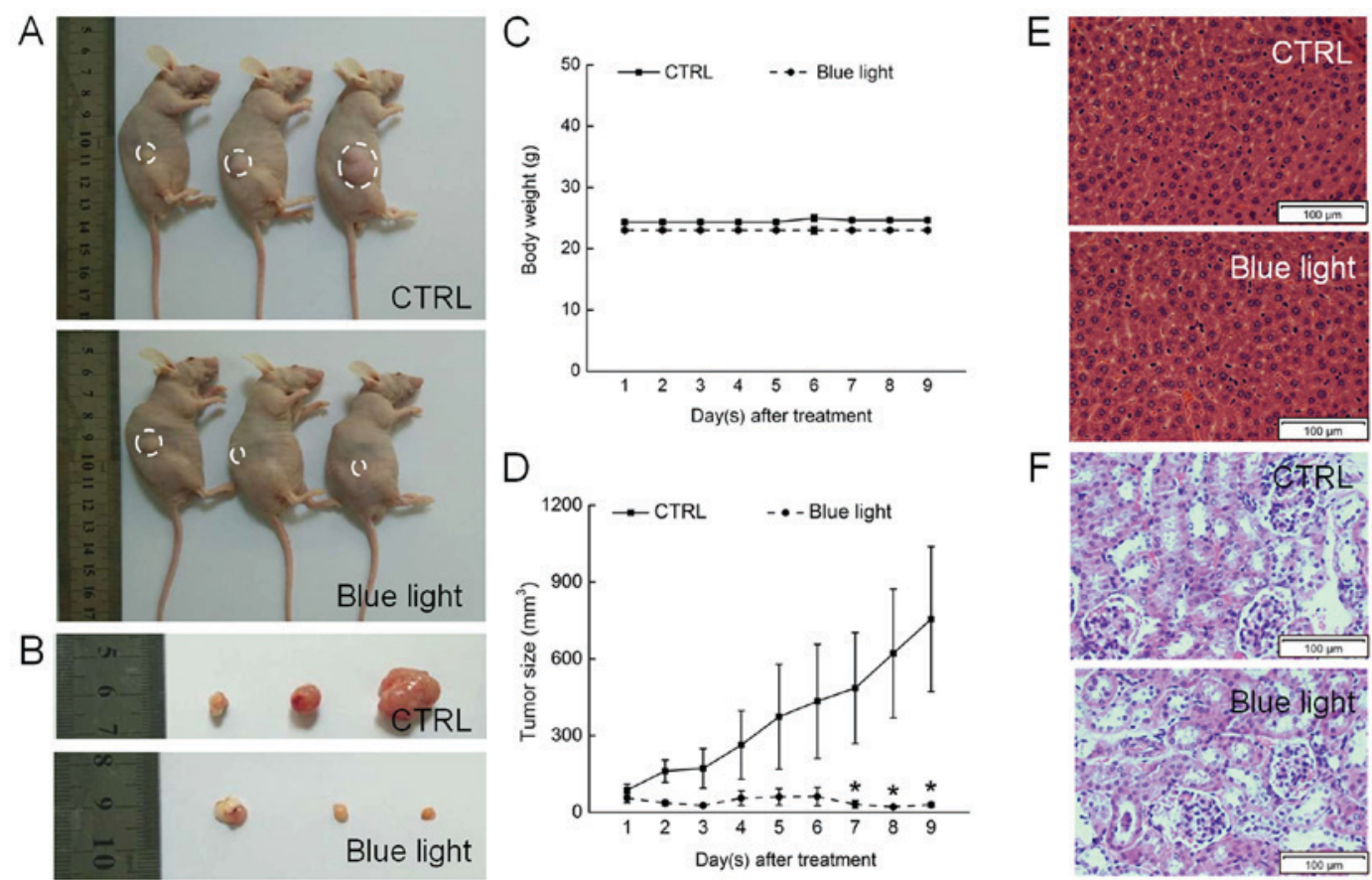

F

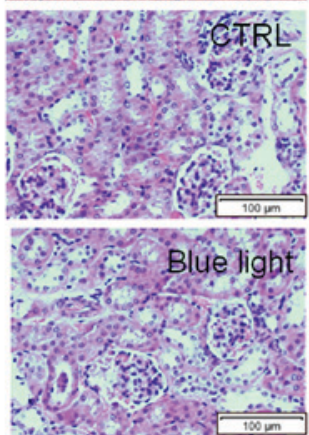

Figure 3. Blue light exposure inhibited HL60-xenografted tumor growth in nude mice. (A) Tumor-possessing nude mice and (B) the excised tumor tissues. (C) Blue light exposure produced no significant effects on body weight. (D) Tumors were measured every day, and the curve of tumor size was expressed as the mean \pm standard deviation ( $\mathrm{n}=3$ ). ${ }^{*} \mathrm{P}<0.05$ vs. CTRL. (E) Histopathological analysis in the liver detected by H\&E staining (magnification, $\mathrm{x} 40$; Scale bars $=100 \mu \mathrm{m}$ ). (F) Histopathological analysis in the kidneys detected by H\&E staining (magnification, $\mathrm{x} 40$; Scale bars=100 $\mu \mathrm{m}$ ). H\&E, hematoxylin and eosin; CTRL, control. 


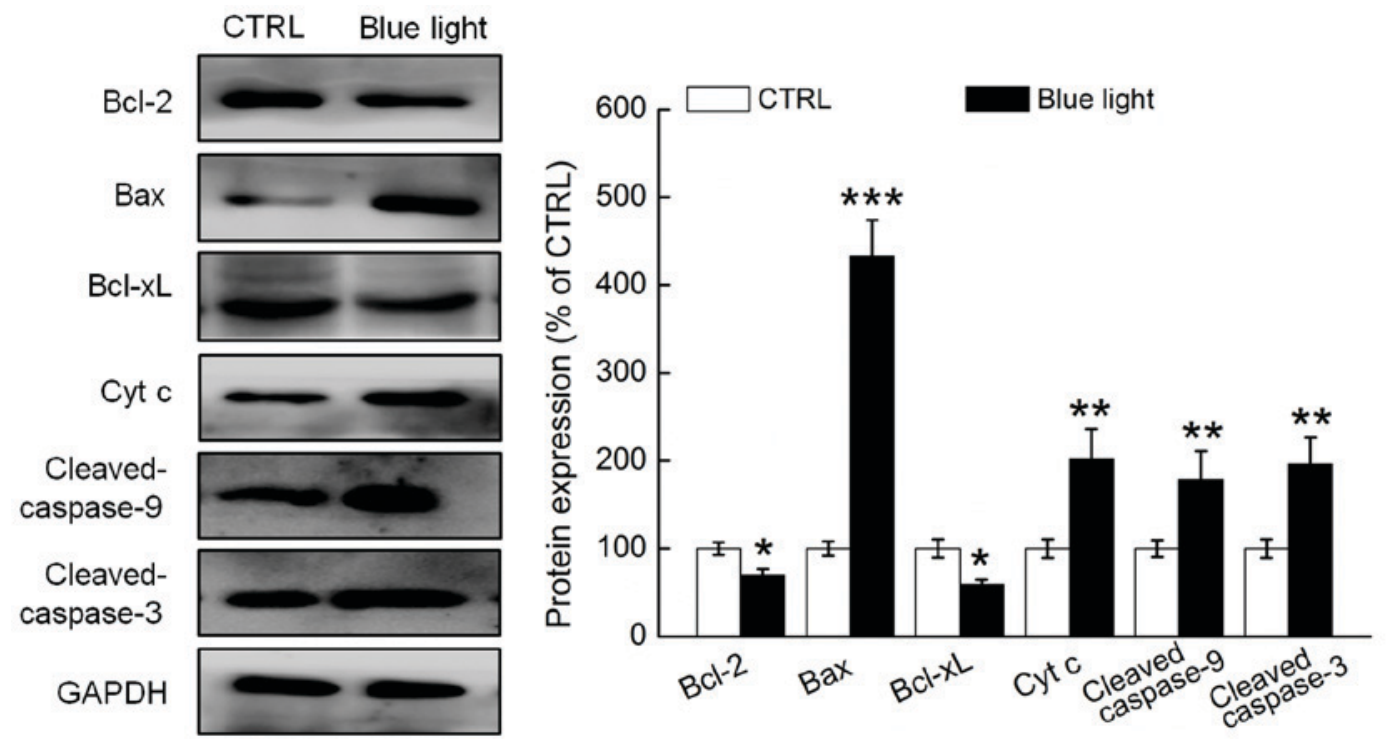

Figure 4. Nine-day exposure of blue light regulated the expressions of pro- and anti-apoptosis proteins in tumor tissues. Quantification data of the protein expressions were normalized to GAPDH, and expressed as a percentage of CTRL and the mean \pm standard deviation $(\mathrm{n}=3)$. ${ }^{*} \mathrm{P}<0.05,{ }^{* *} \mathrm{P}<0.01$ and ${ }^{* * * *} \mathrm{P}<0.001$ vs. control mice. CTRL, control; Bcl-2, B-cell lymphoma 2; xL, extra-long; Bax, Bcl-2-associated X protein; Cyt c, cytochrome $c$.

cell morphology and certain biochemical events associated with apoptosis, is catalyzed by activated caspase-9 (18), and ROS was identified as necessary for the full activation of the caspase cascade (13). Additionally, Bcl-2 family proteins, which serve as biomarkers of mitochondrial function, have become targets for anti-tumor agents. Specifically, the expression levels of Bcl-2 and Bax are used to determine whether the mitochondrial apoptosis pathway has been activated (19), and a heterodimer of $\mathrm{Bcl}-2 / \mathrm{Bax}$ is known to regulate the MMP (20). Our related data further confirm that the pro-apoptotic effects of blue light in HL60 cells may be associated with the mitochondrial pathway.

There are still several limitations in our present study. Firstly, the effects of blue light were not measured in normal cells; however, the histopathological study on livers and kidneys of nude mice has confirmed the safety of blue light therapy. Secondly, although the APL mouse model has been successfully established in our group, it failed to perform in the present study. The tumor bearing mice model established by HL60 cells applied in this experiment has been used for other previous studies (21), which can also certify the pro-apoptotic effects of blue light in human promyelocytic leukemia. Moreover, our present data failed to systemically discuss the correlation between MMP depolarization and ROS production during blue light mediated pro-apoptosis in HL60 cells. Blue light caused the changes on the expression levels of other Bcl-2 family members such as Bim and Bak will be detected in further experiments. N-Acetyl-L-cysteine (NAC), a ROS scavenger, will be applied to investigate its influence on the pro-apoptotic effects of blue light, and the translocation of Cytoplasma $\mathrm{C}$ from mitochondria to cytosol will be detected in cells in our ongoing experiments related to the effects of blue light.

In conclusion, our experimental findings verified the pro-apoptotic effects of blue light in both in vitro and in vivo models. Blue light exposure induced the excess release of LDH and depolarization of the MMP in HL60 cells, suppressed the growth of HL60-xenografted tumors and regulated the expression of both pro- and anti-apoptotic proteins in tumor tissues. Taken together, our data suggest that blue light induces apoptosis in tumor cells via the mitochondrial apoptosis pathway. Our findings provide pharmacological evidence of the potential usefulness of blue light as an adjuvant therapy for leukemia.

\section{Acknowledgements}

Not applicable.

\section{Funding}

This work was supported by the Natural Science foundation of China (Grant No. 81402955).

\section{Availability of data and materials}

All data generated and analysed during the present study are included in this published article.

\section{Authors' contributions}

DW and HL designed the experiments, and wrote and revised the manuscript; JZ, YL and QY performed the experiments and drafted the manuscript; JL and YL analyzed the data.

\section{Ethics approval and consent to participate}

The animal experimental protocol was approved by the Animal Ethics Committee of Jilin University (Jilin, China; no. 2015-003).

\section{Consent for publication}

Not applicable. 


\section{Competing interests}

The authors declare that they have no competing interests.

\section{References}

1. Shakor AB, Atia M, Ismail IA, Alshehri A, El-Refaey H, Kwiatkowska K and Sobota A: Curcumin induces apoptosis of multidrug-resistant human leukemia HL60 cells by complex pathways leading to ceramide accumulation. Biochim Biophys Acta 1841: 1672-1682, 2014.

2. Zhang Y, Zeng C, Lu S, Qin T, Yang L, Chen S, Chen J and $\mathrm{Li}$ Y: Identification of miR-125b targets involved in acute promyelocytic leukemia cell proliferation. Biochem Biophys Res Commun 478: 1758-1763, 2016.

3. Yu M, Guo F, Wang J, Tan F and Li N: A pH-Driven and photoresponsive nanocarrier: Remotely-controlled by near-infrared light for stepwise antitumor treatment. Biomaterials 79: 25-35, 2016.

4. You J, Shao R, Wei X, Gupta S and Li C: Near-infrared light triggers release of Paclitaxel from biodegradable microspheres: Photothermal effect and enhanced antitumor activity. Small 6: 1022-1031, 2010

5. Penjweini R, Loew HG, Breit P and Kratky KW: Optimizing the antitumor selectivity of PVP-Hypericin re A549 cancer cells and HLF normal cells through pulsed blue light. Photodiagnosis Photodyn Ther 10: 591-599, 2013.

6. Zhuang J, Liu J, Liu Y, Li H, Wang D and Teng L: Enhanced proliferation inhibition of HL60 cells treated by synergistic all-trans retinoic acid/blue light/nanodiamonds. RSC Adv 7: 38895-38901, 2017.

7. Mohan V, Agarwal R and Singh RP: A novel alkaloid, evodiamine causes nuclear localization of cytochrome-c and induces apoptosis independent of p53 in human lung cancer cells. Biochem Biophys Res Commun 477: 1065-1071, 2016.

8. Indran IR, Tufo G, Pervaiz S and Brenner C: Recent advances in apoptosis, mitochondria and drug resistance in cancer cells. Biochim Biophys Acta 1807: 735-745, 2011.

9. Kim BM, Choi YJ, Han Y, Yun YS and Hong SH: N, N-dimethyl phytosphingosine induces caspase-8-dependent cytochrome $\mathrm{c}$ release and apoptosis through ROS generation in human leukemia cells. Toxicol Appl Pharmacol 239: 87-97, 2009.

10. Wen Q, Zhang X, Cai J and Yang PH: A novel strategy for real-time and in situ detection of cytochrome c and caspase-9 in Hela cells during apoptosis. Analyst 139: 2499-2506, 2014.
11. Jia G, Wang Q, Wang R, Deng D, Xue L, Shao N, Zhang Y, Xia X, Zhi F and Yang Y: Tubeimoside-1 induces glioma apoptosis through regulation of $\mathrm{Bax} / \mathrm{Bcl}-2$ and the ROS/Cytochrome C/Caspase-3 pathway. Onco Targets Ther 8: 303-311, 2015.

12. Di Giovanni S, Mirabella M, Papacci M, Odoardi F, Silvestri G and Servidei S: Apoptosis and ROS detoxification enzymes correlate with cytochrome c oxidase deficiency in mitochondrial encephalomyopathies. Mol Cell Neurosci 17: 696-705, 2001.

13. McManus MJ,Murphy MPandFranklinJL:Mitochondria-derived reactive oxygen species mediate caspase-dependent and -independent neuronal deaths. Mol Cell Neurosci 63: 13-23, 2014.

14. Hsing CH, Chen CL, Lin WC and Lin CF: Propofol treatment inhibits constitutive apoptosis in human primary neutrophils and granulocyte-differentiated human HL60 cells. PLoS One 10: e0129693, 2015.

15. Mayer B and Oberbauer R: Mitochondrial regulation of apoptosis. News Physiol Sci 18: 89-94, 2003.

16. Degli Esposti M and McLennan H: Mitochondria and cells produce reactive oxygen species in virtual anaerobiosis: Relevance to ceramide-induced apoptosis. FEBS Lett 430: 338-342, 1998

17. Green DR and Reed JC: Mitochondria and apoptosis. Science 281: 1309-1312, 1998.

18. Hu Q, Wu D, Chen W, Yan Z and Shi Y: Proteolytic processing of the caspase- 9 zymogen is required for apoptosome-mediated activation of caspase-9. J Biol Chem 288: 15142-15147, 2013.

19. Raisova M, Hossini AM, Eberle J, Riebeling C, Wieder T, Sturm I, Daniel PT, Orfanos CE and Geilen CC: The Bax/Bcl-2 ratio determines the susceptibility of human melanoma cells to CD95/Fas-mediated apoptosis. J Invest Dermatol 117: 333-340, 2001.

20. Ding J, Mooers BH, Zhang Z, Kale J, Falcone D, McNichol J, Huang B, Zhang XC, Xing C, Andrews DW and Lin J: After embedding in membranes antiapoptotic Bcl-XL protein binds both Bcl-2 homology region 3 and helix 1 of proapoptotic Bax protein to inhibit apoptotic mitochondrial permeabilization. J Biol Chem 289: 11873-11896, 2014.

21. Zhu B, Zhang H and Yu L: Novel transferrin modified and doxorubicin loaded Pluronic 85/lipid-polymeric nanoparticles for the treatment of leukemia: In vitro and in vivo therapeutic effect evaluation. Biomed Pharmacother 86: 547-554, 2017.

This work is licensed under a Creative Commons Attribution-NonCommercial-NoDerivatives 4.0 International (CC BY-NC-ND 4.0) License. 